\title{
In vivo anti-carcinogenic property of a formulated citrus peel extract
}

\author{
Michiko Suzawa ${ }^{1}$, Limin Guo ${ }^{2}$, Min-Hsiung $\mathrm{Pan}^{3}$, Chi-Tang $\mathrm{Ho}^{4}$, Shiming $\mathrm{Li}^{4,5} *$
}

${ }^{1}$ Miyauchi Citrus Research Center, Ltd., 324-10 Shigoka-Machi, Takasaki, Gunma 370-0845, Japan; ${ }^{2}$ Xinjiang Academy of Agricultural Sciences, Urumqi, Xinjiang 830091, China; ${ }^{3}$ Department of Seafood Science, National Kaohsiung Marine University, Kaohsiung 811, Taiwan; ${ }^{4}$ Department of Food Science, Rutgers University, New Brunswick, NJ 08901, USA; ${ }^{5}$ Hubei Key Laboratory of Economic Forest Germplasm Improvement and Resources Comprehensive Utilization, Huanggang Normal University, Huanggang, Hubei, China

*Corresponding author: Shiming Li, PhD, Department of Food Science, Rutgers University, New Brunswick, NJ 08901, USA; Huanggang Normal University, Huanggang, Hubei, China

Submission date: November 26, 2013; Acceptance date: March 24, 2014; Publication date: March 31, 2014

\section{ABSTRACT}

Background: Cancer is one of the two leading fatal diseases humans face. Synthesized drugs available for cancer intervention have many limitations in applications and effectiveness and are often associated with serious of side effects, which can further damage the patients' quality of life. Recently, the development of natural-product-based and therapeutically sound anti-cancer agents have gained popularity in the fields of functional and medical foods, which may exhibit advantages of minimal toxicity and multiple active molecular components. Citrus peel or its extract has been reported to have potent pharmacological activities and health benefits because of abundant flavonoids present in citrus fruits, particularly in the peels.

Results: The results of these studies demonstrated the efficacy of Gold Lotion (GL), an extract of multiple varieties of citrus peels that contains abundant flavonoids, including a high percentage of polymethoxylflavones (PMFs), which can protect against skin cancer, colon cancer, and prostate cancer in mice. These results are clearly promising and warrant a human trial with GL in future studies.

Summary: Briefly, these data have demonstrated that GL is efficacious in preventing and treating cancer in several model systems. This review summarizes the results of currently available data regarding the in vivo anti-cancer activity of GL, and identifies opportunities for subsequent human clinical trials to assess preventive and therapeutic effects in the near future.

Keywords: gold lotion, citrus peel extracts, skin cancer, colon cancer, prostate cancer 


\section{BACKGROUND}

Citrus peel is a rich source of flavonoids and almost the sole source of polymethoxyflavones. Traditional use of citrus peel, such as aged tangerine peels, can be traced back to 10 century BC. However, the exploration of the biological activity of citrus peel started a decade or so ago, and since then, a plethora of its biological functions important to health and diseases have been identified ${ }^{1,2}$. The biological functions of citrus peels investigated include inflammation inhibition, cancer prevention and intervention, hypolipidemia, and regulation of metabolic syndrome. In this paper, we summarize the in vivo anti-cancer effect of a specifically formulated product: Gold Lotion or GL, which is comprised of extracts from the peels of six citrus fruits produced in Japan. In the characterization of the phytochemical composition of GL, we have found abundant flavonoids and a high content of polymethoxyflavones, which consists of a total concentration of more than $550 \mathrm{ppm}$ of flavonoids in the formulated liquid form of $\mathrm{GL}^{3}$.

The anti-cancer activity of GL was previously evaluated on three mouse models, namely, skin cancer ${ }^{4}$, colon cancer ${ }^{3}$, and prostate cancer ${ }^{5}$. In a mouse skin cancer model, the topical application of GL effectively inhibited molecular markers of skin inflammation and attenuated DMBA/TPA-induced skin tumor formation by reducing the tumor incidence and tumor multiplicity of papillomas at 20 weeks. These in vivo data indicate that GL can be an effective anti-tumor agent capable of preventing inflammation associated skin tumorigenesis ${ }^{4}$.

In the model of AOM (azoxymethane)-induced colonic tumorigenesis, oral feeding of GL decreased the number of aberrant crypt foci (ACF), and the proportion of large ACF in colonic tissues of mice. Both gene and protein expression of iNOS and COX-2 were suppressed by GL treatment. The in vivo data have revealed for the first time that the citrus peel extract, GL, is an effective anti-tumor agent mechanistically down-regulating the protein levels of iNOS, COX-2, ODC, VEGF, and MMP-9 in colonic tissues of mice, suggesting a mechanism underlying its therapeutic function ${ }^{3}$.

Prostate cancer is one of the most prevalent male diseases ${ }^{6}$. While mortality due to prostate cancer has decreased recently owing to improved early diagnosis and selection of more efficacious medicinal substances ${ }^{7}$, it remains one of the most common malignancies with a high incidence, an increase in hormone-resistant types, and metastasis ${ }^{8}$. In searching for efficacious and low toxicity medications against prostate cancer, we evaluated GL in a xenograft mouse model. Our data demonstrated that treatment with GL by either intraperitoneal (i.p.) injection or oral administration dramatically reduced the weight and volume of the prostate tumor without observed toxicity. This inhibitory effect was accompanied by a down-regulation of proteins associated with inflammation (iNOS and COX-2), metastasis (MMP-2 and MMP-9), angiogenesis (VEGF), and induction of apoptosis in prostate tumors. Encouraging results as shown above suggest that GL be an effective anti-cancer agent and provide a potential new therapeutic option for prostate cancer treatment ${ }^{5}$.

\section{METHODS}

This section is a brief summary of the materials and methods used in each anti-cancer study. The detailed procedures including materials and methods were described in previous published research papers as cited herein ${ }^{3-5}$. 


\subsection{Experiments in mouse skin cancer ${ }^{4}$}

Animal handling. Female ICR mice (5-6 weeks old) were housed in a controlled atmosphere $\left(25 \pm 1{ }^{\circ} \mathrm{C}, 50 \%\right.$ relative humidity) with a 12-h light/12-h dark cycle. The dorsal skin of each mouse was shaved with surgical clippers before the application of tested compound. GLand TPA were dissolved in $200 \mu \mathrm{L}$ of acetone and applied topically to the shaved area of each mouse.

Measurement of epidermal hyperplasia. The measurement of epidermal thickness is described in previous paper ${ }^{4}$.

Western blot analysis. The mice were treated topically with GL on their shaved backs 30 min prior to $10 \mathrm{nmol}$ of TPA treatment. The rest of the detailed procedure is illustrated in previous paper ${ }^{4}$.

Reverse transcription-polymerase chain reaction (RT-PCR) ${ }^{4}$. Total RNA was isolated from scraped colon mucosa and changes in the steady-state concentration of mRNA in iNOS, and COX-2 and $\beta$-actin were assessed by RT-PCR. Total of $2 \mu \mathrm{g}$ RNA was transcribed into cDNA using SuperScript II Reverse Transcriptase to a final volume of $20 \mu \mathrm{L}$. RT reactions were performed at $50{ }^{\circ} \mathrm{C}$ for $50 \mathrm{~min}$ then $70{ }^{\circ} \mathrm{C}$ for $15 \mathrm{~min}$. The thermal cycle conditions were initiated at $95{ }^{\circ} \mathrm{C}$ for $1 \mathrm{~min}$, and 30 cycles of amplification $\left(94{ }^{\circ} \mathrm{C}\right.$ for $30 \mathrm{~s}, 58{ }^{\circ} \mathrm{C}$ for $25 \mathrm{~s}$, and $72{ }^{\circ} \mathrm{C}$ for $1 \mathrm{~min}$ ), followed by extension at $72{ }^{\circ} \mathrm{C}$ for $3 \mathrm{~min}$. The complementary DNA was amplified by PCR with the primers iNOS, COX-2, and $\beta$-actin, as defined in previous published procedures. PCR products were analyzed by $1 \%$ agarose gel and visualized by ethidium bromide staining.

Two-stage skin carcinogenesis model $^{4}$. Twelve female ICR mice were given commercial rodent pellets and fresh tap water ad libitum. The dorsal region of each mouse was shaved two days prior to initiation. Six week old mice were treated with $200 \mathrm{nmol}$ of DMBA in $200 \mu \mathrm{L}$ acetone and the control group only received $200 \mu \mathrm{L}$ of acetone. One week after initiation, the mice were topically treated with $200 \mu \mathrm{L}$ acetone or with TPA $(5 \mathrm{nmol}$ in $200 \mu \mathrm{L}$ acetone) twice a week for 20 weeks. For the two GL-treated groups, the mice were applied with GL(100 $\mu \mathrm{L}) 30 \mathrm{~min}$ before each TPA treatment. Tumors of at least $1 \mathrm{~mm}^{2}$ of diameter in an electronic digital caliper were counted and recorded twice weekly, and the diameter of skin tumors were measured at the same time.

\subsection{Experiments in mouse colon cancer $^{3}$}

Six week old male ICR mice were randomly distributed into four groups, and groups 2, 3, and 4 were given AOM at a dose of $5 \mathrm{mg} / \mathrm{kg}$ via an i.p. injection twice a week for 2 weeks, and group 1 was received saline by i.p. injection. All groups of mice were fed with the daily control diets. Groups 3 and 4 were orally administered with 100 and $200 \mu \mathrm{L}$ of GL five times a week, respectively. Groups 1 and 2 were given $200 \mu \mathrm{L}$ of phosphate-buffered saline (PBS). The diet intake of the animals was monitored daily. After 6 weeks of feeding, mice were sacrificed by $\mathrm{CO}_{2}$ asphyxiation for evaluation of ACF in colonic tissues. The liver, kidneys, and spleen were removed and weighed. The entire colon was excised, cut longitudinally, rinsed with PBS, and fixed flat between sheets of filter paper with $10 \%$ buffered formalin overnight. 


\subsection{Experiments in mouse prostate cancer ${ }^{5}$}

In vivo tumor xenograft study. Male Balb/c nude mice of 3-4 weeks old were used in this experiment. Prostate cancer PC-3 cells (American Type culture Collection, Manassas, VA, $3 \times 10^{6}$ ) in $0.2 \mathrm{~mL}$ PBS were injected subcutaneously between the scapulae of each nude mouse. After transplantation, tumor size was measured using calipers, and the tumor volume was estimated according to the following formula: tumor volume $\left(\mathrm{mm}^{3}\right)=\mathrm{L} \times \mathrm{W}^{2} / 2$, where $\mathrm{L}$ is the length and $\mathrm{W}$ the width. Once tumors reached a mean size of $50-100 \mathrm{~mm}^{3}$, animals were subjected to i.p. treatment or oral administration of GL. In i.p. treatment, animals received i.p. injections either $50 \mu \mathrm{L}$ of PBS or two dosages of GL, $25 \mu \mathrm{L}$ or $50 \mu \mathrm{L}$, respectively, five times per week for 23 days. Animals were orally administered with either $200 \mu \mathrm{L}$ of sterile water or GL $(50 \mu \mathrm{L}$ or $100 \mu \mathrm{L})$ five times a week for 21 days. At the end of experiment, tumors were excised, weighed, and subjected to western blot analysis. Average tumor volume and tumor weight ratios of each group were measured at the end of the experiment and represent the mean \pm SD.

Western blot analysis. Protein analyses were performed, and the primary antibodies used were iNOS, MMP-2, MMP-9, PCNA, and VEGF polyclonal antibodies, as well as COX-2 and caspase- 3 monoclonal antibody. The detailed protocol and antibody information was described in the original research paper ${ }^{5}$.

\section{RESULTS AND DISCUSSION}

\subsection{Inhibition of mouse skin cancer by $\mathrm{GL}^{4}$}

\subsubsection{Inhibition of iNOS and COX-2 expression by GL in mouse skin}

In the TPA-induced acute inflammatory response model in mouse skin, treatment with TPA induced both iNOS and COX-2 expression in mouse skin, as measured by western blot and semi-quantitative PCR analysis. In this model, a topical application of 100or $200 \mu \mathrm{L}$ GL 30 min prior to TPA treatment resulted in an apparent dose-related reduction of iNOS gene expression, as well as a dramatic reduction of TPA-induced expression of these inflammation-associated proteins and ODC protein, which has been associated with tumor promotion.

\subsubsection{GL Inhibition of TPA-induced epidermal hyperplasia in mouse skin}

A dose of $10 \mathrm{nmol}$ TPA application led to marked edema and an increase in epidermal thickness $(5.4 \pm 1.9 \mu \mathrm{m}$ in acetone group versus $13.2 \pm 0.7 \mu \mathrm{m}$ in TPA group). However, , treatment with 100 and $200 \mu \mathrm{L}$ of GL prior to TPA application significantly reduced epidermal thickness in a dose-dependent manner $(10.1 \pm 1.3 \mu \mathrm{m}$ in $100 \mu \mathrm{L}$ GL group and 8.9 $\pm 1.4 \mu \mathrm{m}$ in $200 \mu \mathrm{L}$ GL group).

\subsubsection{Inhibition of tumor promoting effects in two-stage carcinogenesis skin model}

The mouse skin model of multistage carcinogenesis can be divided into three distinct stages: initiation, promotion, and progression ${ }^{9}$ [8], which serves as a major in vivo model for studying the sequential and stepwise evolution of the cancer process by chemical and physical carcinogens. The steps of this standard model include topical application of a single sub-carcinogenic dose of skin carcinogen, such as DMBA that causes irreversible DNA damage; then repeated application of promoters, most commonly phorbol esters, such as TPA to induce cell proliferation and inflammation ${ }^{10}$. This promotes the selective clonal expansion 
of initiated epidermal cells and leads to the formation of multiple squamous papillomas.

In the two-stage skin carcinogenesis model to assess the antitumor-promoting potential of GL, the untreated positive control group of mice had $16 \pm 3$ papillomas per mouse and a $100 \%$ incidence of skin tumors at 20 weeks, whereas no tumors were observed in the negative control group following acetone application. However, when $100 \mu \mathrm{L}$ GL was applied to the shaven backs of mice $30 \mathrm{~min}$ prior to each TPA application, the average number of papillomas per mouse was $12 \pm 4$ (25\% reduction compared to positive group). In addition, the tumor incidence was found to be $100 \%$ in positive group whereas GL-treated group showed significantly decreased at $81 \%$. Moreover, the weight of the tumors in the positive group was $0.31 \pm 0.12 \mathrm{~g}$, but significantly lower in GL-treated group $(0.11 \pm 0.07 \mathrm{~g})$.

\subsubsection{Tumor size decrease and inhibition of angiogenetic molecule expression in mouse skin tumors.}

The number of papillomas ( $\geqq 5 \mathrm{~mm}$ in diameter) per mouse was significantly decreased in the GL treated group ( $9 \pm 2$ in positive group and $6 \pm 1$ in GL group). In addition, in the positive control group in which mouse skin tumors were initiated by DMBA and promoted by TPA for 20 weeks, the protein expression of ODC, COX-2, and VEGF (vascular endothelial growth factor) was apparently increased compared to healthy skin tissue. However, treatment with $100 \mu \mathrm{L}$ of GL resulted in a strong reduction of ODC, COX-2, and VEGF protein levels in skin tumors. ODC is involved in cancer proliferation and both COX-2 and VEGF contribute to angiogenesis, hence we suggest that GL reduced tumor size by inhibiting the tumor growth and angiogenesis.

\subsection{Colon cancer inhibition by $\mathbf{G L}^{3}$}

In the AOM model, the progression is from ACF (aberrant crypt foci) to adenomas and to invasive cancers. During this progression, formation of ACF in the early stage is considered to be a histological biomarker of colonic tumor development ${ }^{11}$. Large ACF are dysplastic, with histological features of micro adenomas that are associated with an increased risk for malignant progression ${ }^{12}$. In the efficacy study of GL against colon cancer, the number of ACFs in GL $(200 \mu \mathrm{L})$ treated mice is $12 \pm 2(P<0.05)$, remarkably lower than non-GL-, but AOM-treated group of mice $(16 \pm 2)$. Surprisingly, the number of larger ACFs was targeted first and reduced to $16 \pm 3(100 \mu \mathrm{L} \mathrm{GL})$ and $15 \pm 2(200 \mu \mathrm{L} \mathrm{GL})$ respectively, compared to 25 \pm 3 ACFs in the AOM-treated positive control group $(P<0.05)^{3}$.

The development of colorectal cancer involves various genetic and molecular changes in cell proliferation, inflammation, resistance to apoptosis, angiogenesis, and metastasis ${ }^{13}$. Over expression of iNOS and COX-2 enzymes contribute to the promotion of tumorigenesis by induction of inflammation, abnormal cell proliferation, and decreasing apoptosis ${ }^{14}$. Thus, the effect of GL on AOM-induced inflammatory (iNOS and COX-2), proliferative (ODC) and angiogenic (VEGF and MMP-9) molecule expression in mouse colon, was assessed. As shown in Figure 1A, expression of inflammatory enzymes iNOS and COX-2 were increased in AOM-treated group. However, oral administration of GL at dosages of $100 \mu \mathrm{L}$ and $200 \mu \mathrm{L}$ dose-dependently decreased AOM-induced iNOS and COX-2 protein level in mouse colon compared with the AOM-treated positive control group. Also, the gene expression of both $i$ NOS and COX-2 genes were markedly reduced in GL-treated groups compared to AOM-treated group (Figure 1B). Interestingly, the gene expression of COX-2 showed a complete decrease in $200 \mu \mathrm{L}$ of GL treated group (Figure 1B). These results suggested that 
the anti-inflammatory activity of GL might be one of the mechanisms for its chemopreventive activity in inhibiting AOM-induced colonic tumorigenesis. In addition, the proliferative marker ODC, the metastasis marker MMP-9, and the angiogenic molecule VEGF, which were up-regulated in AOM-treated mouse colon, were apparently reduced in a dose-related fashion by oral administration of GL. Therefore, the suppression of ACF formation might be through GL inhibiting cellular proliferation and angiogenesis in the colonic mucosa ${ }^{3}$.These results suggest that the mechanism of in vivo chemopreventive efficacy of GL includes anti-inflammation, anti-proliferation, and anti-angiogenesis in AOM-induced colonic tumorigenesis.

\section{Figure 1A}

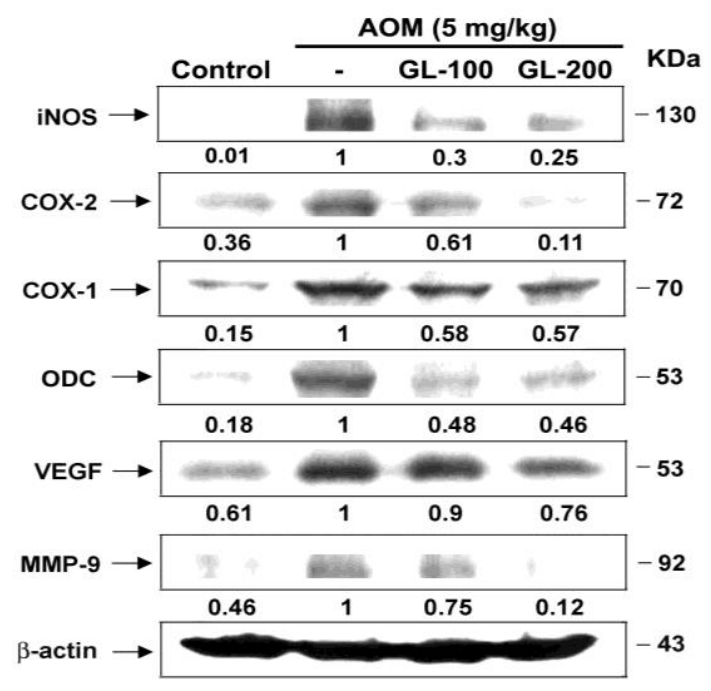

Figure 1B

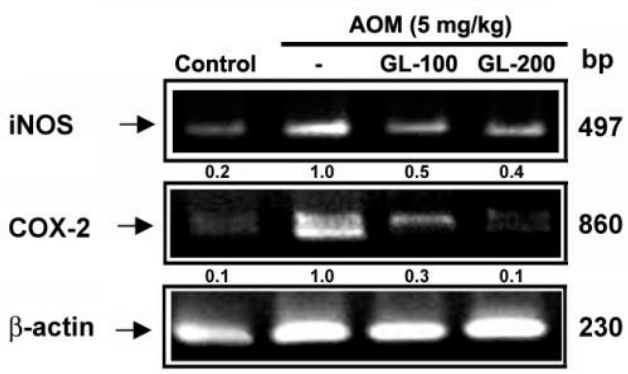

Figure $\mathbf{1}^{\mathbf{3}}$. Inhibitory effects of oral administration of GL on AOM-induced protein levels in colorectal tissue. (A) Protein lysates from normal colonic mucosa and ACF of each group were extracted and subjected to Western Blot analysis for iNOS, COX-2, COX-1, ODC, VEGF and MMP-9 protein levels. $\square$-actin as an internal control. The western blot is a representative of at least three independent experiments. Quantification of iNOS, COX-2, COX-1, ODC, VEGF and MMP-9 protein expression was normalized to $\square$-actin using a densitometer. (B) Effect of oral administration of GL on AOM-induced inos and cox-2 gene expression in colorectal tissue. RNA from normal colonic mucosa and ACF of each group were extracted and subjected to RT-PCR analysis for inos and cox-2 gene expression.

\section{3 prostate cancer ${ }^{5}$}

The human prostate tumor xenograft model has been widely used in cancer therapy research and anti-cancer drug development ${ }^{15}$. To evaluate potential therapeutic effects on prostate cancer, GL was tested in immune-deficient mice bearing human prostate cancer cell line PC-3 tumor xenografts. After the establishment of palpable tumors, animals received i.p. injections of $25 \square \mathrm{L}$ and $50 \square \mathrm{L}$ GL five times per week for 23 days. An unexpectedly potent inhibition of PC-3 xenograft tumor formation was observed by i.p. injection of GL at $25 \mu \mathrm{L}$. The prostate xenograft tumor was almost completely eliminated in the group treated with $50 \mu \mathrm{L}$ of GL(Figure 2). 


\section{Figure 2A}

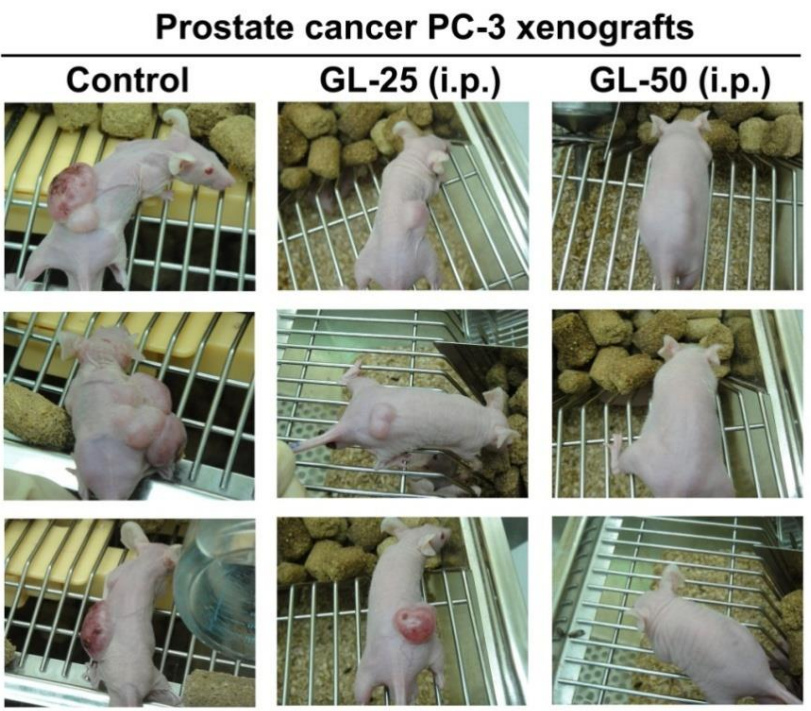

Figure 2B

Figure 2C
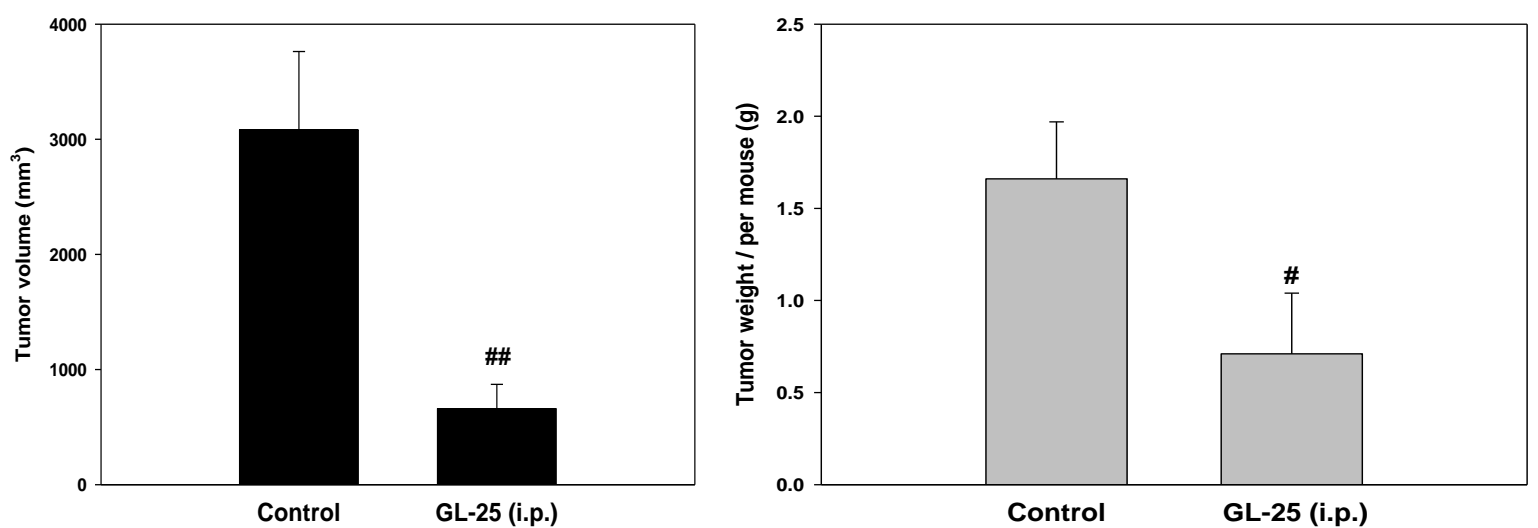

Figure 2. The growth of PC-3 tumor xenografts in nude mice was reduced by i.p. treatment with GL. (A) Whole body imaging of PC-3 tumor-bearing mice from control and GL group. (B) Average tumor weight and (C) tumor volume were measure at the end of experiment. Five samples were analyzed in each group, and values represent the mean $\pm \mathrm{SD} .{ }^{\#} P<0.01$ and ${ }^{\# \#} P<0.001$, compared with control group.

Consistent with this observation, both tumor weight and tumor size were markedly decreased in the group treated with $25 \mu \mathrm{L}$ of GL. Mice in the control group showed an average tumor weight of $1.66 \pm 0.31 \mathrm{~g}$, whereas this was significantly lower in the group treated with $25 \mu \mathrm{L}$ GL i.p., at $0.71 \pm 0.33 \mathrm{~g}(P<0.01)$ (Figure 2B). Similarly, the average tumor volume was significantly lower in the group treated with $25 \square \mathrm{L}$ GL compared with that of the control group $\left(659.6 \pm 211.1 \mathrm{~mm}^{3}\right.$ vs. $3082.0 \pm 680.4 \mathrm{~mm}^{3}$ respectively; Figure 1C). Based on these results, tumor weight and tumor volume were dramatically inhibited by i.p. injection of GL in the PC-3 xenograft model $^{5}$.

Therapeutic effects of oral dosing with GL were also evaluated in this model. Animals were treated orally with $50 \square \mathrm{L}$ or $100 \square \mathrm{L}$ of GL in two groups five times per week for 21 days. At the end of the study, the body weight in each group was not significantly different 
(Figure 3A): positive group: $20.41 \pm 2.11 \mathrm{~g} ; 50 \mu \mathrm{L}$ GL group, $21.22 \pm 0.53 \mathrm{~g}$; and $100 \mu \mathrm{LGL}$ group, $21.34 \pm 0.90 \mathrm{~g}$.

Figure 3A

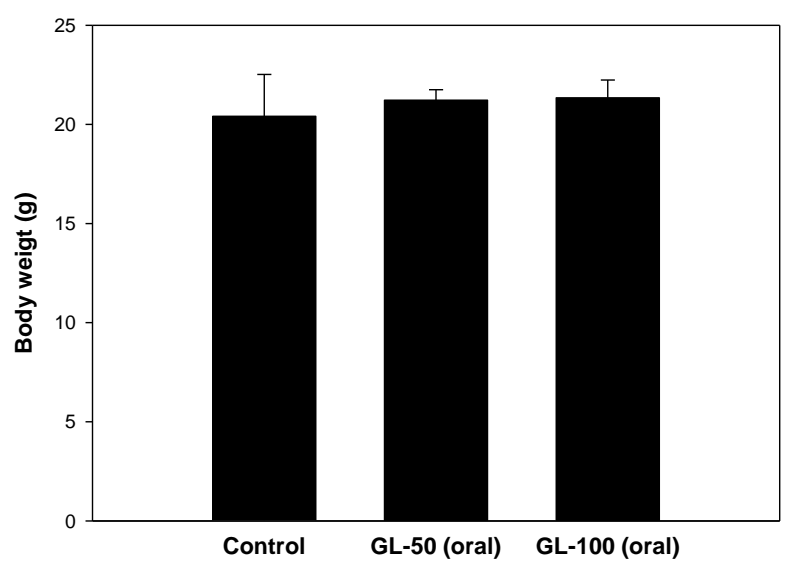

Figure 3B

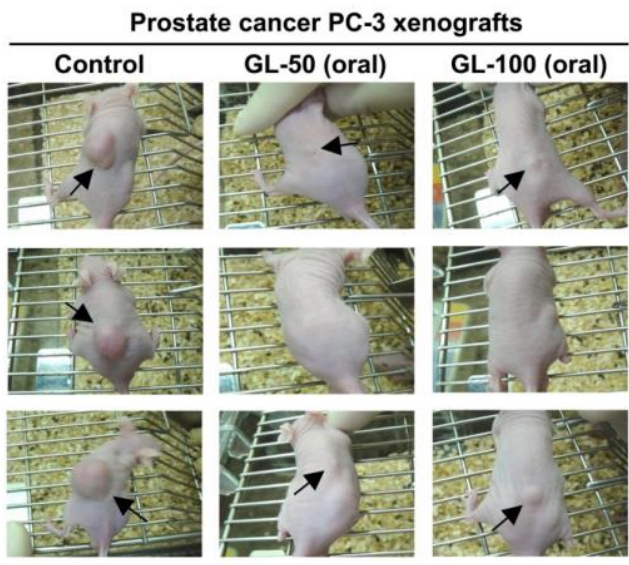

Figure 3C

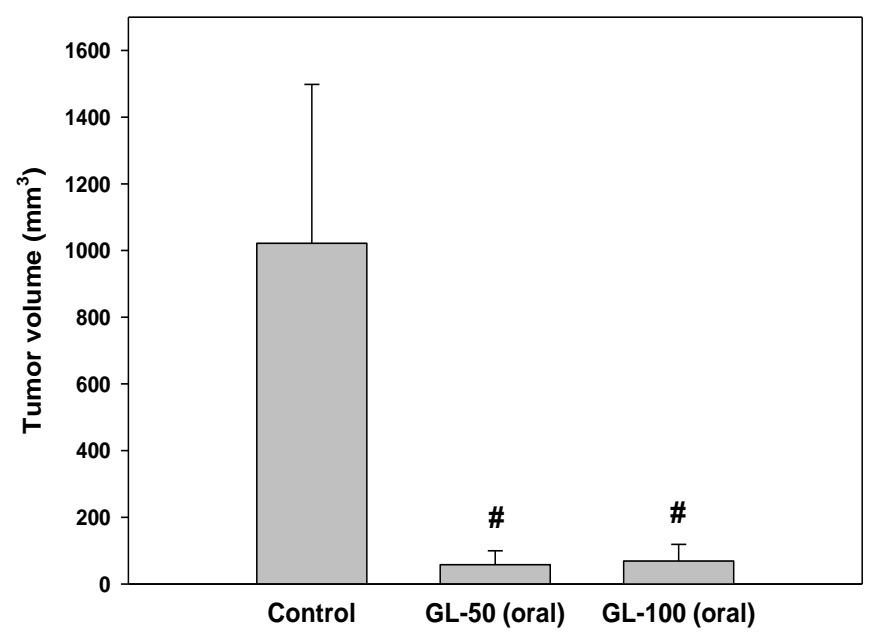

Figure 3. Oral administration of GL suppressed the growth of PC-3 tumor xenografts in nude mice. (A) Body weight and (B) representative photo images of PC-3 tumor-bearing mice from each group at day 21. (C) Tumor volume were measure at the end of experiment. Five samples were analyzed in each group, and values represent the mean \pm SD. Significantly different at ${ }^{\#} P<0.01$ compared to control group.

Figure 3B shows the in vivo macrograph of the PC-3 tumor-bearing mice. After 21 days, nude mice bearing prostate tumor xenografts were visible in control group (arrow). Furthermore, a marked inhibition of PC-3 xenograft tumor number and size was observed by oral GL treatment. The average tumor size in positive group was $1021.9 \pm 476.2 \mathrm{~mm}^{3}$ whereas in the orally GL treated group, both $50 \mu \mathrm{L}$ and $100 \mu \mathrm{L}$, dramatically reduced tumor size to $57.7 \pm 42.0 \mathrm{~mm}^{3}$ and $69.1 \pm 9.9 \mathrm{~mm}^{3}$, respectively (Figure 3C). No dose-response between GL oral feeding of $50 \mu \mathrm{L}$ and $100 \mu \mathrm{L}$ group was observed. These results provided evidence that oral administration of GL also exerted anti-prostate cancer activity in vivo. 
Thus we can conclude that GL may serve as a novel chemopreventive and therapeutic agent for prostate cancer, regardless of administration method, either i.p. injection or oral administration $^{5}$.

\section{CONCLUSION}

In summary, these in vivo studies provide compelling evidence that GL is a potent anti-cancer agent in cancers of skin, colon, and prostate. The therapeutic mechanism may include inhibition of inflammation, proliferation and angiogenesis, and induction of apoptosis. A characteristic and unusual feature of GL is the high concentration of a diverse assortment of PMFs and other flavonoids. In view of other studies examining the impact of individual PMFs and related flavonoids ${ }^{1}$, it is possible that the biological properties of GL summarized here are due to an additive or synergistic interaction of the complex mixture of phytochemicals, which may provide enhanced anti-cancer efficacy. The detailed mechanisms by which these PMFs and flavonoids in GL exert optimal benefits remain to be further elucidated. However, the evidence presented in this review illustrates that GL could prove to be an effective anti-cancer agent, especially against skin, colon, and prostate cancer and encourage future research to evaluate its efficacy in human clinical trials.

Abbreviations: ACF, aberrant crypt foci; AOM, azoxymethane; CRC, colorectal cancer; COX-2, cyclooxygenase-2; DMBA, 7,12-dimethylbenz(a)anthracene; GL, gold lotion; ICR, Institute of Cancer Research; iNOS, inducible nitric oxide synthase; NO, nitric oxide; MMPs, matrix metallopeptidases; ODC, ornithine decarboxylase; PCNA, proliferating cell nuclear antigen; PCR, polymerase chain reaction; PMFs, polymethoxylflavones; TPA, 12-O-tetradecanoylphorbol 13-acetate; VEGF, vascular endothelial growth factor.

Acknowledgements: The authors thank Nancy E. Rawson, Ph.D. for editorial assistance.

Authors' Contributions: All authors contributed to this study.

\section{REFERENCES:}

1. Li S, Pan MH, Lo CY, Tan D, Wang Y, Shahidi F, Ho CT: Chemistry and health effects of polymethoxyflavones and hydroxylated polymethoxyflavones. J. Functional Foods. 2009, 1:2-12.

2. Manthey JA, Grohmann K, Guthrie N: Biological properties of citrus flavonoids pertaining to cancer and inflammation. Curr. Med. Chem. 2001, 8(2):135-153, 2001.

3. Lai CS, Li S, Liu CB, Miyauchi Y, Suzawa M, Ho CT, Pan MH: Effective suppression of azoxymethane-induced aberrant crypt foci formation in mice with citrus peel flavonoids. Mol. Nutr. Food Res. 2013, 57:551-555.

4. Pan MH, Li S, Lai CS, Miyauchi Y, Suzawa M, Ho CT: Inhibition of citrus flavonoids on 12-O-tetradecanoylphorbol 13-acetate-induced skin inflammation and tumorigenesis in mice. Food Sci. Huamn Wellness. 2012, 1:65-73.

5. Lai CS, Li S, Miyauchi Y, Suzawa M, Ho CT, Pan MH: Potent anti-cancer effects of citrus peel flavonoids in human prostate xenograft tumors. Food \& Function. 2013, 4(6):944-949.

6. Obertova Z, Brown C, Holmes M, Lawrenson R: Prostate cancer incidence and 
mortality in rural men--a systematic review of the literature. Rural. Remote. Health. 2012, 12 (2):2039.

7. Hanley JA: Mortality reductions produced by sustained prostate cancer screening have been underestimated. J. Med. Screen. 2010, 17 (3):147-151.

8. Shen MM, Shen C: Molecular genetics of prostate cancer: new prospects for old challenges. Genes Dev. 2010, 24 (18):1967-2000.

9. Wu X, Pandolfi PP: Mouse models for multistep tumorigenesis. Trends Cell Biol. 2001, 11 (11):S2-S9.

10. Hennings H, Glick AB, Greenhalgh DA, Morgan DL. Strickland JE, Tennenbaum T, Yuspa SH: Critical aspects of initiation, promotion, and progression in multistage epidermal carcinogenesis. Proc. Soc. Exp. Biol. Med. 1993, 202 (1):1-8.

11. Takahashi, M, Wakabayashi, K: Gene mutations and altered gene expression in azoxymethane-induced colon carcinogenesis in rodents. Cancer Sci. 2004, 95 (6): 475-480.

12. Otori, K, Sugiyama, K, Hasebe, T, Fukushima, S, Esumi, H: Emergence of adenomatous aberrant crypt foci (ACF) from hyperplastic ACF with concomitant increase in cell proliferation. Cancer Res. 1995, 55 (21):4743-4746.

13. Pan MH, Lai CS, Wu JC, Ho CT: Molecular mechanisms for chemoprevention of colorectal cancer by natural dietary compounds. Mol. Nutr. Food Res. 2011, 55 (1):32-45.

14. Bing RJ, Miyataka M, Rich KA, Hanson N, Wang X, Slosser HD, Shi SR I: Nitric oxide, prostanoids, cyclooxygenase, and angiogenesis in colon and breast cancer. Clin. Cancer Res. 2001, 7 (11):3385-3392.

15. Priolo C, Agostini M, Vena N, Ligon AH, Fiorentino M, Shin E, Farsetti A, Pontecorvi A, Sicinska E, Loda M: Establishment and genomic characterization of mouse xenografts of human primary prostate tumors. Am. J. Pathol. 2010, 176 (4):1901-1913. 